\title{
Rumo à Otimização de Operadores sobre UDF no Spark*
}

\author{
João Antonio Ferreira ${ }^{1}$, Fábio Porto ${ }^{2}$, Rafaelli Coutinho ${ }^{1}$, Eduardo Ogasawara ${ }^{1}$ \\ ${ }^{1}$ CEFET/RJ - Centro Federal de Educação Tecnológica Celso Suckow da Fonseca \\ ${ }^{2}$ LNCC - Laboratório Nacional de Computação Científica \\ joao.paranadacm.org, fportodlncc.br \\ rafaelli.coutinho@cefet-rj.br, eogasawara@ieee.org
}

\begin{abstract}
Large-scale data analysis has gained much importance in the scientific community due to the Big Data phenomenon. In this context, user-defined functions (UDFs) are commonly implemented in frameworks such as Apache Spark to enable large-scale data analysis. However, the use of UDF brings challenges in optimization of execution as they are opaque. This work proposes a method of optimizing data analysis workflows supported by UDF on Apache Spark. This method is based on SparkSQL's Catalyst API and Scala language macros.
\end{abstract}

Resumo. A análise de dados em larga escala tem ganhado muita importância na comunidade científica devido ao fenômeno do Big Data. Neste contexto, funções definidas pelo usuário (UDF) são, comumente, implementadas em frameworks como Apache Spark para viabilizar a análise de dados em larga escala. No entanto, o uso de UDF traz desafios no processo de otimização de execução pois são opacas. Este trabalho propõe um método de otimização de workflows de análise de dados apoiadas em UDF sobre o Apache Spark. Tal método é baseado na API Catalyst do SparkSQL e em macros da linguagem Scala.

\section{Introdução}

A resolução de diversos problemas científicos é computacionalmente intensiva e otimizações voltadas às plataformas de hardware específicas (GPU e FPGA) e algoritmos e métodos matemáticos utilizados não são suficientes devido o aumento da quantidade de fontes de dados. Para se conduzir as análises de dados neste contexto, surgiram soluções para processamento de grandes volumes de dados escaláveis horizontalmente, tal como o modelo MapReduce, implementado em frameworks como Apache Spark [Zaharia et al., 2016].

Funções definidas pelo usuário (UDF) são comumente usadas nesses frameworks de modo a viabilizar a análise de dados em larga escala em experimentos científicos. Apesar de sua importância, o uso de UDF traz desafios no processo de otimização de execução, devido a dificuldade de se estabelecer semântica clara sobre seus comportamentos que possuem códigos relacionados ao domínio. Com a falta de conhecimento sobre a

* Os autores agradecem à FAPERJ, à CAPES e ao CNPq pelo financiamento do trabalho. 
semântica da operação, o Spark é incapaz de otimizar a execução [Armbrust et al., 2015]. Este trabalho propõe um método para otimização das execuções de workflows de análise de dados, que possam ser escritas como UDF sobre o Apache Spark. Como primeiro estudo, avaliou-se a viabilidade do processo de otimização da execução de mapeamentos (operador map) operando sobre UDF usando a API Catalyst do Spark para abordar o problema da execução de atividades restritas (Constrained Activity) ${ }^{1}$ [Ogasawara et al., 2011]. Uma avaliação preliminar aponta a viabilidade da abordagem.

Além desta introdução, o trabalho está organizado em mais três seções. Na seção 2, são apresentados os conceitos gerais necessários para o entendimento do problema e a abordagem adotada sob a forma de prova de conceito. A seção 3 traz a avaliação preliminar que corrobora com a hipótese. Por fim, a seção 4 apresenta as conclusões.

\section{Prova de conceito}

O Spark é um framework que possibilita a execução das tarefas paralelizáveis de forma distribuída em máquinas multi-core ou clusters YARN/Mesos/Kubernetes, com ênfase no processamento em pipeline das atividades que compõem um dataflow [Zaharia et al., 2016]. Ele foi adotado para apoiar os usuários na execução de workflow de análise de dados com UDF em ambientes de processamento distribuído em larga escala [Ferreira et al., 2017]. A vantagem desta abordagem é deixar a responsabilidade sobre a complexidade do modelo de execução para o Spark. Isso diferencia a proposta de Ferreira et al. [2017] dos outros sistemas de gerenciamento de workflow (SGW). Desta forma, o foco se restringe a anotação semântica de UDF que tanto estabelece a relação de consumo e produção de ativações [Ogasawara et al., 2011] quanto na associação com a proveniência para prover informação necessária a otimização do workflow.

Nesta prova de conceito foi realizada uma análise exploratória das APIs do $\mathrm{Ca}$ talyst pertencente ao módulo SparkSQL e dos seus pontos de extensão. O SparkSQL usa o componente Catalyst para otimizar a geração de código em tempo de execução à partir de expressões e comandos SQL, e os pontos de extensão do Spark possibilitam a personalização do ambiente com o uso de novas regras para otimização de dataflow de acordo com o domínio do problema.

Na álgebra de workflow, o operador wMap é definido: $T \leftarrow w M a p(A, R)$, onde $A$ é a atividade que produz uma única tupla na relação de saída $T$ para cada tupla consumida na relação de entrada $R$. Os esquemas de $R$ e $T$ podem ser diferentes num caso mais geral. Portanto, é possível observar que a semântica do operador wMap é compatível com seu análogo map do SparkSQL [Ogasawara et al., 2011; Armbrust et al., 2015]. Considere o seguinte exemplo especificado pela álgebra de workflow: $T \leftarrow w \operatorname{Map}(B, w \operatorname{Map}(A, R))$. Ao implementar este dataflow no Spark, o Catalyst do SparkSQL usará a regra CollapseProject na fase de otimização. Esta regra junta as duas expressões dos dois map em uma, executando $A$ e $B$ em pipeline. Isso pode ser indesejado no caso em que estas atividades forem escritas em código nativo e usarem muitos recursos do hardware. Isto caracteriza, segundo Ogasawara et al. [2011], a existência de uma atividade restrita (Constrained Ac-

\footnotetext{
${ }^{1}$ Nas aplicações científicas é muito comum invocar programas com implementações paralelas que usam todos os núcleos (cores) disponíveis em um nó do cluster. Devido a essa característica, a ativação de uma atividade restrita bloqueia todos os núcleos, inibindo a utilização por outras ativações. Ogasawara et al. [2011] chama este fenômeno de atividade restrita (Constrained Activity).
} 
tivity), onde uma atividade, regida por um operador wMap e executada em um dataflow, pode consumir muitos recursos computacionais impedindo que outras atividades também regidas por um operador wMap possam ser executadas em pipeline. Para solucionar isso, Ogasawara et al. [2011] propõem a criação de uma barreira forçando a materialização do resultado intermediário entre a execução das atividades.

Com o objetivo de otimizar este tipo de workflow, foi criada uma classe ${ }^{2}$ escrita em linguagem Scala para funcionar como ponto de extensão e otimizar operadores map. Além dos pontos de extensão do SparkSQL, esta classe usa as funcionalidades quasiquotes e macros da linguagem Scala para atuar no dataflow antes mesmo de passar o plano lógico da query ao Catalyst. Ela consiste de uma case class do Scala e é responsável por transformar planos lógicos por meio de otimizações baseadas em transformações algébricas. O objetivo desta classe é incluir uma barreira de materialização entre dois operadores map adjacentes. Neste caso, isso só é possível se os operadores atuarem sobre UDF anotadas com semântica de utilização excessiva de recursos em tempo de execução, informações estas obtidas da proveniência.

\section{Avaliação Preliminar}

Nesta seção, procurou-se avaliar a capacidade do Spark em otimizar um dataflow com UDF anotada com informações sobre uso excessivo de recursos usando o operador wMap, como ilustrado abaixo em código Scala:

myDataset.map(heavyUDF1(some, parameters)). map(heavyUDF2(param, ...))

O Spark combina, por padrão, os processamentos dessas duas UDF em pipeline, pois desconhece a semântica da operação executada e os recursos computacionais consumidos. Caso estas UDF sejam escritas em código nativo, cada uma delas pode consumir todos os recursos de thread ou memória do processador de forma que não possam ser invocadas em pipeline. Conforme já mencionado, isto caracteriza uma atividade restrita (Constrained Activity) e a solução é a criação de uma barreira forçando a materialização do resultado entre a avaliação das duas UDF.

A anotação semântica pode ser feita via sufixo indicando a quantidade de memória RAM utilizada em média ou o número de threads/cores do processador, ou ambos. Esta anotação é usada na fase inicial para dividir o workflow original em dois segmentos e ordená-los antes de passar ao Catalyst para que seja feita as outras otimizações possíveis em cada um dos segmentos. As materializações no Spark podem ser provocadas por operadores collect aplicados no dataset. Esta abordagem foi escolhida, por modificar apenas a representação do dado, preservando o esquema. O importante é que a criação da barreira não modifica a semântica do dataflow original.

Para uma avaliação preliminar, a criação de barreira foi aplicada sobre o workflow mostrado na Figura 1(a). Nele, tem-se uma relação R0 onde são aplicados dois operadores map em sequência. O primeiro operador rege a udfA que após anotação semântica se transformou em udfA_M3G. O segundo operador rege a udfB que anotado se transformou em udfB_M3G. O sufixo M3G significa que o processo externo invocado pela UDF usa

\footnotetext{
${ }^{2} \mathrm{O}$ código fonte Scala pode ser acessado em https://github.com/joao-parana/wff-catalyst
} 
em média 3GB de memória RAM para cada tupla consumida. Supondo que o subsistema de proveniência tenha informado que o sistema sofrerá com problemas de desempenho ou confiabilidade nestas condições, o dataflow pôde ser modificado para materializar o resultado do primeiro map. A Figura 1(b) mostra esta situação. Uma relação R1 foi criada com o propósito de materializar o resultado intermediário para que o Spark não realize a otimização padrão para dois operadores map adjacentes. Com isso, o dataflow deixou de ser executado em pipeline permitindo sua finalização, o que não seria possível na versão não original, pois o programa terminaria logo no início por falta de recursos computacionais disponíveis.

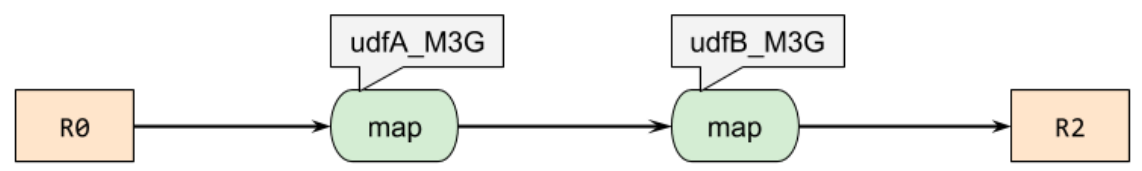

(a)

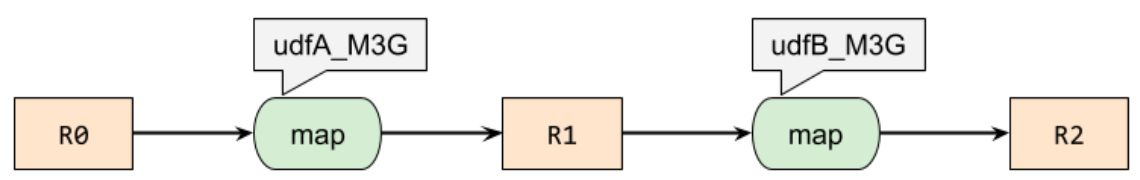

(b)

Figura 1. workflow antes (a) e depois (b) da inclusão da barreira.

\section{Considerações finais}

Em Ferreira et al. [2017] foi abordado a possibilidade de usar o Spark como base para a implementação de um framework de análise de dados usando a álgebra de workflow para facilitar o trabalho dos cientistas. Este presente trabalho aponta que esta abordagem é viável e que é possível criar implementações de otimizações arbitrárias incluindo UDF com o uso dos pontos de extensão disponíveis no SparkSQL. Isso inclui o caso da atividade restrita (Constrained Activity) com atividades legadas escritas em código nativo (C++, Fortran, Python, Cython, R, etc.) [Ogasawara et al., 2011].

\section{Referências}

Armbrust, M., Xin, R., Lian, C., Huai, Y., Liu, D., Bradley, J., Meng, X., Kaftan, T., Frankliny, M., Ghodsi, A., and Zaharia, M. (2015). Spark SQL: Relational data processing in spark. In Proceedings of the ACM SIGMOD International Conference on Management of Data, volume 2015-May, pages 1383-1394.

Ferreira, J., Gaspar, D., Monteiro, B., Silva, A. B., Porto, F., and Ogasawara, E. (2017). Uma Proposta de Implementação de Álgebra de Workflows em Apache Spark no Apoio a Processos de Análise de Dados. In Brazilian e-Science Workshop.

Ogasawara, E., de Oliveira, D., Valduriez, P., Dias, J., Porto, F., and Mattoso, M. (2011). An algebraic approach for data-centric scientific workflows. In Proceedings of the VLDB Endowment, volume 4, pages 1328-1339.

Zaharia, M., Franklin, M. J., Ghodsi, A., Gonzalez, J., Shenker, S., Stoica, I., Xin, R. S., Wendell, P., Das, T., Armbrust, M., Dave, A., Meng, X., Rosen, J., and Venkataraman, S. (2016). Apache spark: A unified engine for big data processing. Communications of the ACM, 59(11):56-65. 